\title{
Study of Tea Digitized Chromatographic Fingerprint Spectra Using Micellar Electrokinetic Chromatography
}

\author{
SONG, Guan-Qun ${ }^{a, b}$ (末冠群) \\ LIN, Jin-Ming ${ }^{\bullet}, a($ 林金明 $)$ \\ QU, Feng ${ }^{a}$ (屈锋) \\ DONG, Wen-Ju ${ }^{b}$ (董文举) \\ ${ }^{a}$ Research Center for Eco-Environmental Sciences, Chinese Academy of Sciences, Beijing 100085, China \\ ${ }^{b}$ College of Chemistry and Environmental Science, Henan Normal University, Xinxiang, Henan 453002, China
}

\begin{abstract}
This paper described the principle of digitized chromatographic fingerprint spectrum and established digitized chromatographic fingerprint spectra of ten brands of Chinese famous tea by the micellar electrokinetic chromatography. This work was done using a $25 \mathrm{mmol} \cdot \mathrm{L}^{-1}$ sodium dodecylsulfate in a $20 \mathrm{mmol} \cdot \mathrm{L}^{-1}$ borate (pH 7.0) solution as running buffer, $20 \mathrm{kV}$ applied potential and detection at $280 \mathrm{~nm}$. The chromatographic fingerprint spectra were digitized by the relative retention value $(\alpha)$ and the relative area $\left(S_{\mathrm{r}}\right)$, and were analyzed to identify the tea samples. In the absence of the standard samples, the present method was easy setup and inexpensive, and provided the applicable information for the quality assessment of teas.
\end{abstract}

Keywords tealeaves, micellar electrokinetic chromatography, digitized chromatographic fingerprint spectrum, quality assessment

\section{Introduction}

Tea is the most widely consumed beverage in people's life. The heightened popularity of this beverage throughout the world in recent years may be due in part to the evidence of a relationship between tea consumption and prevention of certain forms of human disease. The main components of tea are polyphenolic compounds, commonly known as catechins, which represent a group of compounds belonging to the flavonoid family. These compounds are widely distributed in the tea leaves and constitute up to $30 \%$ of the dry leaf weight. ${ }^{1}$ Much interest has been focused on catechins, not only for their antioxidant activity, ${ }^{2}$ but also for their known antimutagenic and antitumorigenic properties. ${ }^{3,4}$ Because of the above characteristics, the intake of tea becomes more and more. According to data published by the Tea Council in the United Kingdom, global production is about 3 million tones each year, ${ }^{5}$ the majority of which come from China. Therefore, the tea assessment is highly desirable, and many methods have been developed for the determination of catechins, e. g. , TLC, ${ }^{6}$ HPLC, ${ }^{7}$ HPLC-MS,${ }^{8} \mathrm{CZE}^{9}$ and MEKC. ${ }^{10}$ However, these methods are always used for assessing the tea quality or establishing the tea criterion by one or two components as the guideline such as (-)-epicatechin, $(-)$-epigallocatechin, ( - )-epigallocatechin gallate. Therefore, they can not totally reflect the quality of tea. It is indispensable to establish a simple and convenient method for assessing the quality of tea by measuring several components of tea simultaneously.

The composition of tealeaves depends on many factors, e.g., agronomic condition, leaf age, storage time, the degree of fermentation, etc. Therefore, the components of tealeaves are very complex. The studies indicate that in the same chromatographic conditions, different brands of tea result in different chromatograms, even though from the same brand, there have great difference between chromatograms because of resources and period. Based on these reasons, we establish the digitized chromatographic fingerprint spectrum consisted of relative-retention time $(\alpha)$ and corresponding peak area $\left(S_{t}\right)$ using the micellar electrokinetic chromatography. The present method can transfer chromatograms to datasheets, resulting in reducing the effect of operational factors and the fluctuation of peak location and improving the comparability among samples. The most important of all, this method can still obtain reliable results without standard samples.

\section{Experimental}

\section{Apparatus}

All experiments were carried out with a lab-built (capillary electrophorecis) system equipped with a high voltage power supply (Beijing New Tech. Inc., China), a UV spectrometer (Jasco, Japan) and a plexiglass box in which the buffer and sample reservoirs were. A fused-silica capillary (Yongnian, China) with a total length of 60 $\mathrm{cm}$ and an I.D. of $50 \mu \mathrm{m}$ was used. A detection window was created at $45 \mathrm{~cm}$ from the capillary inlet by removing

* E-mail: jmlin@mail.rcees.ac.cn

Received January 22, 2003; revised April 8, 2003; accepted June 9, 2003.

Project supported by the National Natural Science Fundation for Distinguished Young Scholars of China (No. 20125514) and the National Natural Science Foundation of China (Nos. 20275043 and 50273046). 
the polyimide coating. HW chromatography software (Qianpu Inc., China) was used for both the operation of the detector and data processing. THZ-82 constant-temperature shaker (Changzhou Inc., China) was used to dispose the sample.

\section{Reagents}

All reagents were of analytical-reagent grade. Sodium dodecylsulfate (SDS) was obtained from Wako Pure Chemival Industries Ltd (Tokyo, Japan). All solutions were prepared with water, which was deionized using a Milli-Q system with $0.2-\mu \mathrm{m}$ fiber filter $(18.3 \mathrm{M} \Omega$, Barnstead, USA). The tea samples and their resources were listed in Table 1.

\section{Analytical conditions}

A $25 \mathrm{mmol} \cdot \mathrm{L}^{-1} \mathrm{SDS}$ in a $20 \mathrm{mmol} \cdot \mathrm{L}^{-1}$ borate $(\mathrm{pH}$ 7.0) solution was used as running solution. A potential of $20 \mathrm{kV}$ was applied and the detection was performed by UV absorption at $280 \mathrm{~nm}$. The samples were injected by 1.5 $\mathrm{kPa}$ pressure for $10 \mathrm{~s}$.

The capillary was conditioned prior to use by flushing for 10 min with $1.0 \mathrm{~mol} \cdot \mathrm{L}^{-1} \mathrm{HCl}, 2 \mathrm{~min}$ with water, 10 min with $1.0 \mathrm{~mol} \cdot \mathrm{L}^{-1} \mathrm{NaOH}, 2 \mathrm{~min}$ with water and finally $10 \mathrm{~min}$ with buffer. In order to increase the reproducibility of the migration time, between runs the capillary was rinsed consecutively with $1.0 \mathrm{~mol} \cdot \mathrm{L}^{-1} \mathrm{HCl}(2 \mathrm{~min})$, water $(1 \mathrm{~min})$ and finally with buffer $(2 \mathrm{~min})$.

\section{Preparation of tea sample solution}

$1.000 \mathrm{~g}$ of tealeaves were weighted and placed into a $250 \mathrm{~mL}$ of beaker, adding $100 \mathrm{~mL}$ of deionized hot water at $90{ }^{\circ} \mathrm{C}$, stirring for $10 \mathrm{~min}$ by a constant temperature shaker at $90^{\circ} \mathrm{C}$. When cooling at about $70^{\circ} \mathrm{C}, 20 \mathrm{~mL}$ of tea infusion filtered with common filter paper was diluted 10 times with $0.1 \%$ metaphosphoric acid. After passing through the $0.45-\mu \mathrm{m}$ membrane filter, the filtrates were used as samples.

\section{Results}

\section{Optimization of the separation condition}

Watababe et al.$^{10}$ reported that catechins were separated in SDS-borate-phosphate buffer solution at $\mathrm{pH}=7.0$.
However, in the present work, it was found that this solution caused a higher value of current, unacceptable Joule heating and peak broading, being presumably due to the fact that the alteration of electroosmotic flow affected the separation with different capillary. In order to achieve the rapid and valid separation, the buffer system was optimized. The results showed that the viscosity of the solution was increased with the increasing of the concentration of SDS and borate, resulting in the decreased velocity of electroosmotic flow and longer separation time. Therefore, a $25 \mathrm{mmol} \cdot \mathrm{L}^{-1} \mathrm{SDS}$ in a solution of $20 \mathrm{mmol} \cdot \mathrm{L}^{-1}$ borate $(\mathrm{pH}=7.0)$ was chosen as the optimum buffer system, resulting in the moderate separation time and the good separation.

Establishment of digitized chromatographic fingerprint spectrum

Under the optimum conditions, the chromatograms of the ten brands of Chinese tea were obtained. As shown in Fig. 1, the chromatograms were characteristic but the quality of tea could not be identified only from them. Thus, it was necessary to establish the digitized chromatographic fingerprint spectra according to the ten chromatograms.

The chromatographic peaks shown in Fig. 1 were dense. One of the peaks was selected as the reference peak, which was characteristic of strong intensity, mid-retention time and common in the ten chromatograms. Thus, based on the reference peak, the relative-retention time $(\alpha)$ can be obtained through the formulation:

$$
\alpha=t_{\mathrm{r}} / t_{\mathrm{r}}
$$

$t_{\mathrm{rs}}$ is absolute retention time of reference peak, $t_{\mathrm{r}}$ is absolute retention time of determined peaks.

The chromatogram of Molihua tea (sample No. III in Table 1) was selected as the standard sample because of its more peaks, stronger peak intensity and representative peak shape. The relative area $\left(S_{\mathrm{r}}\right)$ of determined peak can be obtained according to the total peak area of the standard. The equation was:

$$
S_{\mathrm{r}}=\left(A_{\mathrm{r}} / A_{\mathrm{rs}}\right) \times 100
$$

$A_{\mathrm{rs}}$ was the total peak area of the standard sample, $A_{\mathrm{ri}}$ was the area of the determined peak.

Table 1 Ten brand Chinese teas and their resources for the present experiment

\begin{tabular}{cllcll}
\hline No. & \multicolumn{1}{c}{ Sample } & Source & No. & Sample & Source \\
\hline I & Huangshan maofeng & Anhui & VI & Qijing greentea & Fujian \\
II & Xinyang maojian & Henan & VII & Mingqian luzhen & Fujian \\
III & Molihua tea & Shanghai & VIII & Biluochun & Jiangsu \\
IV & Dahongpao & Fujian & IX & Guihua woolong & Anhui \\
V & Xihu longjing & Zhejiang & X & Tieguanyin & Fujian \\
\hline \hline
\end{tabular}



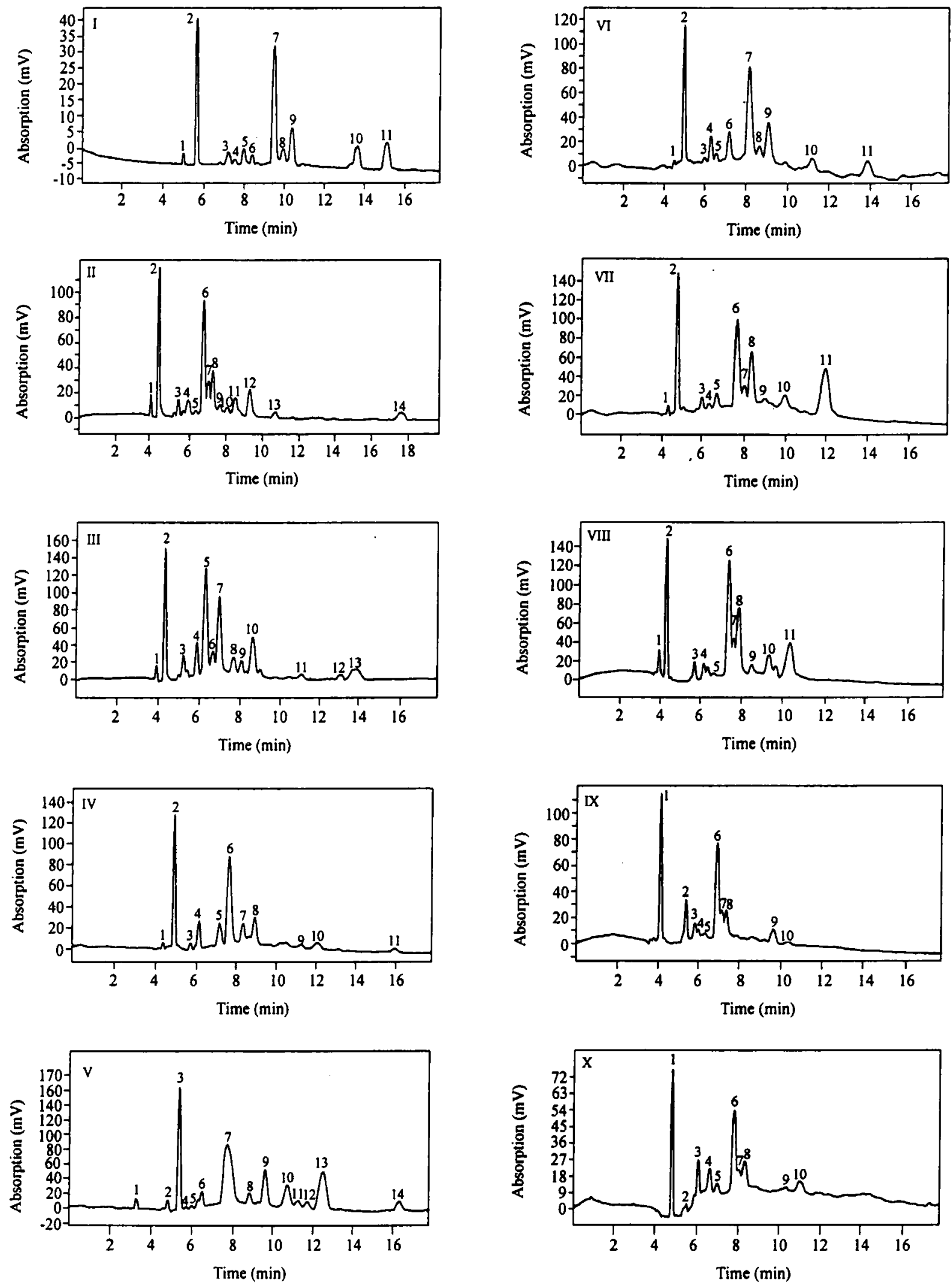

Fig. 1 Chromatograms of the ten brands of Chinese tea. Conditions: capillary, $50 \mu \mathrm{m} \mathrm{i.d.} \times 45 \mathrm{~cm}$; running solution, $25 \mathrm{mmol} \cdot \mathrm{L}^{-1} \mathrm{SDS}$ solution in $20 \mathrm{mmol} \cdot \mathrm{L}^{-1}$ borate buffer $(\mathrm{pH}=7.0)$; applied voltage, $20 \mathrm{kV}$; detection, $280 \mathrm{~nm}$; injection, $1.5 \mathrm{kPa} \times 10 \mathrm{~s}$. (I) Huangshan Maofeng (Anhui); (II) Xinyang maojian (Henan); (III) Molihua tea (Shanghai) as the standard; (IV) Dahongpao (Fujian); (V) Xihu longjing (Zhejiang); (VI) Qijing greentea (Fujian); (VII) Mingqian luzhen (Fujian); (VIII) Biluochun (Jiangsu); (IX) Guihua woolong (Anhui); (X) Tieguanyin (Fujian). 
The digitized chromatographic fingerprint spectrum (Table 2) was finished by establishing a chart, arraying the $\alpha$ value from minimum to maximum, lining out the value of $S_{\mathrm{r}}$ under the corresponding $\alpha$ and then analyzing the $S_{\mathrm{r}}$ with the same $\alpha$ value.

\section{Method validation}

Using the Molihua tea as the sample, the precision and reproducibility of the method were evaluated by carrying out five replicate analyses on the same day. In all cases the value of the relative standard deviation for the retention time and peak area were lower than $1.0 \%$ and $5.0 \%$, respectively. This result indicated that the established digitized chromatographic fingerprint spectrum had well precision and reproducibility.

\section{Superposition ratio of chromatographic peaks}

The superposition ratio was that based on the respec- tive peak number and the mutual peak (the peaks with the same $\alpha$ ) number of the compared two samples, from the view of nature their correlation was studied. The formulation of the superposition ratio $(R)$ was:

$$
R=\frac{2 n}{N} \times 100
$$

$n$ was the mutual peak number of analyzed and standard samples and $N$ was the total peak number of analyzed and standard samples.

The chromatography of the No. III sample was appointed as the standard. The superposition ratio of chromatographic peaks of the ten samples can be obtained. They were $25.0,44.4,100.0,41.7,44.4,41.7$, $41.7,33.3,50.0$ and 60.8 , respectively.

Eight strong chromatographic peaks

Eight strong chromatographic peaks were referred

Table 2 Analysis of chromatographic fingerprint of 10 brand Chinese teas

\begin{tabular}{|c|c|c|c|c|c|c|c|c|c|c|}
\hline \multirow{2}{*}{$a^{b}$} & \multicolumn{10}{|c|}{$S_{\mathrm{r}}^{a}$} \\
\hline & $\mathbf{I}$ & II & III & IV & $\mathbf{V}$ & VI & VII & VIII & $\mathbf{L X}$ & $\mathbf{X}$ \\
\hline 0.526 & 0.24 (1) & & $1.35(1)$ & & $1.42(1)$ & & $2.01(1)$ & $3.33(1)$ & & \\
\hline 0.578 & $3.62(2)$ & $1.32(1)$ & & $0.46(1)$ & & $0.72(1)$ & & $17.2(2)$ & 7.21 (1) & \\
\hline 0.618 & & $9.5(2)$ & $13.3(2)$ & $11.2(2)$ & $1.36(2)$ & $14.1(2)$ & $15.4(2)$ & & $5.56(2)$ & $7.26(1)$ \\
\hline 0.683 & & & & & $17.2(3)$ & & & & & \\
\hline 0.739 & & & & & $0.82(4)$ & $1.93(3)$ & & & & \\
\hline 0.772 & $0.57(3)$ & & & $0.66(3)$ & $1.20(5)$ & $4.93(4)$ & $6.26(3)$ & $2.76(3)$ & $3.31(3)$ & $2.23(2)$ \\
\hline 0.791 & $0.33(4)$ & 2.01 (3) & & $3.43(4)$ & & $2.39(5)$ & & & & \\
\hline 0.838 & $0.82(5)$ & & $3.38(3)$ & & $3.74(6)$ & & $3.11(4)$ & $2.52(4)$ & $2.06(4)$ & $5.52(3)$ \\
\hline 0.875 & $0.46(6)$ & $2.67(4)$ & & & & $9.86(6)$ & $6.31(5)$ & $2.20(5)$ & $2.73(5)$ & $4.22(4)$ \\
\hline 0.939 & & $0.95(5)$ & $5.59(4)$ & $4.81(5)$ & & & & & & $2.52(5)$ \\
\hline 1.000 & $5.45(7)$ & $11.9(6)$ & $20.0(5)$ & $16.1(6)$ & $34.6(7)$ & $21.7(7)$ & $19.3(6)$ & $19.9(6)$ & $9.55(6)$ & $13.1(6)$ \\
\hline 1.046 & $1.04(8)$ & $3.92(7)$ & & & & & $11.5(7)$ & $5.39(7)$ & & \\
\hline 1.061 & & $5.64(8)$ & $5.56(6)$ & & & $4.15(8)$ & & $14.1(8)$ & $3.70(7)$ & $3.70(7)$ \\
\hline 1.110 & $1.98(9)$ & & $18.3(7)$ & $5.55(7)$ & & $12.2(9)$ & $15.5(8)$ & & $4.20(8)$ & \\
\hline 1.143 & & $1.53(9)$ & & & $6.20(8)$ & & & $5.03(9)$ & & \\
\hline 1.174 & & $1.79(10)$ & & $6.95(8)$ & & & $4.99(9)$ & & & \\
\hline 1.224 & & & $5.83(8)$ & & & & & & & \\
\hline 1.254 & & $4.31(11)$ & $4.39(9)$ & & $13.3(9)$ & & $14.0(10)$ & $9.65(10)$ & & \\
\hline 1.374 & & $4.86(12)$ & $10.5(10)$ & & & $5.79(10)$ & & & & $6.95(8)$ \\
\hline 1.403 & $1.44(10)$ & & & & $10.9(10)$ & & & $12.8(11)$ & $3.65(9)$ & $0.83(9)$ \\
\hline 1.460 & & & & $2.20(9)$ & $3.63(11)$ & & & & $1.83(10)$ & \\
\hline 1.518 & & & & & $3.38(12)$ & & & & & \\
\hline 1.588 & $1.95(11)$ & $1.14(13)$ & & $3.12(10)$ & $11.3(13)$ & $5.15(11)$ & $25.8(11)$ & & & \\
\hline 1.768 & & & $2.59(11)$ & & & & & & & \\
\hline 2.086 & & & $2.03(12)$ & $0.93(11)$ & $3.90(14)$ & & & & & \\
\hline 2.205 & & & $7.18(13)$ & & & & & & & $6.26(10)$ \\
\hline 2.617 & & $1.98(14)$ & & & & & & & & \\
\hline $\begin{array}{l}\text { Quantity of } \\
\text { total peaks }\end{array}$ & 11 & 14 & 13 & 11 & 14 & 11 & 11 & 11 & 10 & 10 \\
\hline
\end{tabular}

\footnotetext{
${ }^{a}$ The relative area of determined peak (the number in the parenthesis is corresponded to the peak number): ${ }^{b}$ the relative-retention time.
} 
to the eight peaks with higher value of $S_{\mathrm{r}}$, and the difference of the teas can be compared from the view of quantum. The eight strong chromatographic peaks of the ten samples were in Table 3 .

\section{Discussion}

In this study, in order to get integrated chromatograms, the analytical time was controlled in an hour, while all the analyzed peaks appeared in twenty minutes. From Table 2 it can be seen that the chromatographic peaks were gathered at the value of $\alpha$ from $0.578 \sim 1.110$. One of the peaks, the $\alpha$ value of which was 1.000 , was the common peak of all samples. So this peak as one of the necessary condition was used to identify tea samples. Therefore, there were twelve peaks in all as characteristic peaks to identify the tea samples. Their $\alpha$ values were $0.526,0.578,0.618,0.772,0.838,0.875,1.000$, $1.061,1.110,1.254,1.403$ and 1.588 , respectively.

From the superposition ratio, it was found that the superposition ratios of the other samples were all lower than $50 \%$ except the Nos. 9 and 10 samples. These data indi- cated that the components of tealeaves had great variability. This was mainly due to the great difference in species, resource, age, climate and horticultural conditions (e.g., soil, water, minerals, fertilizers) etc.

From Table 3 it could be found that the peaks of $\alpha$ amount to 1.000 were all in the eight strong peaks with higher content, and the peaks of $\alpha$ amount to 0.618 were the common peak of eight samples, seven of which were in the eight strong peaks. So these two components were the main components of tea. It also could be found that the relative peak areas of the eight strong peaks were all different. Although the same resource, the relative peak areas were different. Take the tea from Fujian for example, the relative peak areas of the four brands of tea were from $51.53 \%$ to $114.07 \%$. Therefore, to some extent the quality of the tea was correlative with the breed.

The above results indicated that the digitized chromatographic fingerprint spectrum reflected the distribution of the components and content of the tealeaves, and the characteristic peaks as the foundation were used to identify the tea. In the absence of the standard, it is feasible to identify the quality of the tea according to the chromatographic fingerprint.

Table 3 Eight strong peaks of chromatographic fingerprint of 10 samples

\begin{tabular}{|c|c|c|c|c|c|c|c|c|c|}
\hline \multirow{2}{*}{$\begin{array}{c}\text { Sample } \\
\text { No. }\end{array}$} & \multicolumn{8}{|c|}{$\alpha^{a}$} & \multirow{2}{*}{$\begin{array}{c}\text { Relative peak } \\
\text { area }(\%)\end{array}$} \\
\hline & 1 & 2 & 3 & 4 & 5 & 6 & 7 & 8 & \\
\hline I & $1.000(7)$ & $0.578(2)$ & $1.110(9)$ & $1.588(11)$ & $1.403(10)$ & $1.046(8)$ & $0.838(5)$ & $0.772(3)$ & 16.87 \\
\hline II & $1.000(6)$ & $0.618(2)$ & $1.061(8)$ & $1.374(12)$ & $1.254(11)$ & $1.046(7)$ & $0.875(4)$ & $0.791(3)$ & 44.81 \\
\hline III & $1.000(5)$ & $1.110(7)$ & $0.618(2)$ & $1.374(10)$ & $2.205(13)$ & $1.224(8)$ & $0.939(4)$ & $1.061(6)$ & 86.26 \\
\hline IV & $1.000(6)$ & $0.618(2)$ & $1.174(8)$ & $1.110(7)$ & $0.939(5)$ & $0.791(4)$ & $1.588(10)$ & $1.460(9)$ & 53.36 \\
\hline $\mathbf{v}$ & $1.000(7)$ & $0.683(3)$ & $1.254(9)$ & $1.588(13)$ & $1.403(10)$ & $1.143(8)$ & $2.086(14)$ & $0.838(6)$ & 101.14 \\
\hline VI & $1.000(7)$ & $0.618(2)$ & $1.110(9)$ & $0.875(6)$ & $1.374(10)$ & $1.588(11)$ & $0.772(8)$ & $1.061(4)$ & 77.88 \\
\hline VII & $1.588(11)$ & $1.000(6)$ & $1.110(8)$ & $0.618(2)$ & $1.254(10)$ & $1.046(7)$ & $0.875(5)$ & $0.772(3)$ & 114.07 \\
\hline VIII & $1.000(6)$ & $0.578(2)$ & $1.061(8)$ & $1.403(11)$ & $1.254(10)$ & $1.046(7)$ & $1.143(9)$ & $0.526(1)$ & 87.40 \\
\hline $\mathbf{X X}$ & $1.000(6)$ & $0.578(1)$ & $0.618(2)$ & $1.110(8)$ & $1.061(7)$ & $1.403(9)$ & $0.772(3)$ & $0.875(5)$ & 39.91 \\
\hline $\mathbf{x}$ & $1.000(6)$ & $0.618(1)$ & $1.374(8)$ & $2.205(10)$ & $0.838(3)$ & $0.875(4)$ & $1.061(7)$ & $0.939(5)$ & 51.53 \\
\hline
\end{tabular}

- The mean results of five times determinations. The number in the parenthesis is corresponded to the peak number.

\section{References}

1 Goto, T.; Yoshida, Y.; Kiso, M.; Nagashima, H. J. Chromatogr. , A 1996, 749, 295.

2 Vinson, J.; Dabbagh, Y.; Serry, M.; Jang, J. J. Agric. Food Chem. 1995, 43, 2800.

3 Zhao, B. Chin. Sci. Bull. 2002, 47, 1206 (in Chinese).

4 Lin, Y.; Juan, I.; Chen, Y.; Liang, Y.; Lin, J. J. $A$ gric. Food Chem. 1996, 44, 1387.
5 Khokhar, S.; Magnusdottir, S. J. Agric. Food Chem. 2002, 50, 565 .

6 Sun, B.; Leandro, C.; Silva, J.; Spranger, I. J. Agric. Food Chem. 1998, 46, 1390.

7 Lee, B.; Ong, C. J. Chromatogr., A 2000, 881, 439.

8 Poon, G. J. Chromatogr. A, 1998, 794, 63.

9 Horie, H.; Mukai, T.; Kohata, K. J. Chromatogr., A 1997, 758, 332.

10 Watanabe, T.; Nishiyama, R.; Yamamoto, A.; Nagai, S.; Terabe, S. Anal. Sci. 1998, 14, 435. 